\title{
„Antrag auf den Aufbau einer Sammlung zur Geschichte der Zuwanderung nach Nordrhein- Westfalen“
}

1 https://www.landtag.nrw.de/portal/WWW/ dokumentenarchiv/ Dokument/MMD145351.pdf (aufgerufen am 10.05.2021).

Während die Bundeskanzlerin Merkel auf Bundesebene im Jahr 2006 den ersten Integrationsgipfel einberief, verabschiedete die Landesregierung von NRW einen Aktionsplan zur Integration. Zu dem 20-Punkte-Plan sollte auch die Einrichtung eines zentralen Archivs zur Erforschung der Migrationsgeschichte des Landes NRW gehören. Bereits am 6. November 2007 hatte die damalige Regierungsfraktion von CDU und FDP einen Antrag zum „Aufbau einer Sammlung zur Geschichte der Zuwanderung nach Nordrhein-Westfalen“ in den nordrhein-westfälischen Landtag eingebracht. ${ }^{1}$ Darin hieß es: „Integration in Gegenwart und Zukunft kann [...] nicht gestaltet werden ohne ein Bewusstsein für die historische Dimension der zugrundeliegenden Zuwanderungsprozesse. Die kritische Reflexion der Vergangenheit ist eine entscheidende Voraussetzung für den Abbau von Vorurteilen und die Stärkung des gesellschaftlichen Zusammenhalts. Vor allem jedoch setzt die unverkrampfte, aber respektvolle Begegnung der Zuwanderer und der aufnehmenden Gesellschaft zunächst eine gefestigte Identität aller Beteiligten voraus. Deshalb bedarf es einer Kultur des Erinnerns, die sowohl den Bezug zum Herkunftsland als auch die spezifischen Lebenssituationen der Zugewanderten heute in Nordrhein-Westfalen in den Blick nimmt."

Die Verfasser*innen dieses Antragstexts argumentierten - erstaunlich genau dem (Selbst-)Verständnis DOMiDs entsprechend -, man solle den Einwander*innen und ihren Nachkommen in der geschichtlichen Überlieferung des Landes auch Identifikationsmomente, gemeinsame Erinnerungsorte bieten. Der Antrag spricht nun selbst von der „nationalen Bedeutung “ des Themas und fordert dazu auf, einen geeigneten Raum für diese (trans-)nationale Erinnerungsarbeit zu finden. Das neue Ruhrmuseum - das ehemalige Ruhrlandmuseum - auf Zeche Zollverein wurde ins Spiel gebracht, dem eine eigene Abteilung zur Zuwanderungsgeschichte 
ins Ruhrgebiet angegliedert werden sollte. Dann kommt im Antrag die Sprache explizit auf DOMiD, das sich als "materielles Gedächtnis der Einwanderer" (Borsdorf) etabliert habe. Es liege daher nahe, den Verein mit dem Aufbau eines solchen zentralen Erinnerungsortes zu betrauen: „In Nordrhein-Westfalen sammelt bereits das Dokumentationszentrum und Museum über die Migration in Deutschland e.V. (DOMiD) in Köln Dokumente und Materialien zur Migrationsgeschichte in Deutschland seit den 1950er-Jahren. Diese Sammlung ist in ihrem Fortbestand zu sichern und auszubauen, ohne dabei einzelne Aspekte und Interessensgruppen einseitig hervorzuheben. Außerdem ist sicherzustellen, dass sie weiter be-

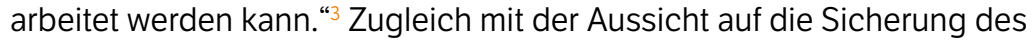
Bestandes gab die Landesregierung DOMiD also gewissermaßen den Arbeitsauftrag an die Hand, der Viel(ge)schichtigkeit der Einwanderungsgeschichte politisch wie historisch Gerechtigkeit widerfahren zu lassen und den Sammlungsbestand auch zukünftig konsequent zu erweitern: „Die Zuwanderungsgeschichte Nordrhein-Westfalens ist eine Geschichte von großer Heterogenität. Dies betrifft nicht nur die diversen Herkunftsländer, sondern auch die unterschiedlichen historischen Situationen und Bedingungen von Migration und Integration zwischen freiwilliger Zuwanderung, Zwangsarbeit, Flucht und Heimatvertreibung sowie staatlich organisiertem Anwerben von Arbeitskräften. Die Landesregierung wird daher aufgefordert, dafür Sorge zu tragen, dass die Zuwanderungsgeschichte Nordrhein-Westfalens in ihrer ganzen Breite und Vielfältigkeit erfasst wird. Es geht um eine Sammlung der Migrationskultur aller Zuwanderinnen und Zuwanderer."

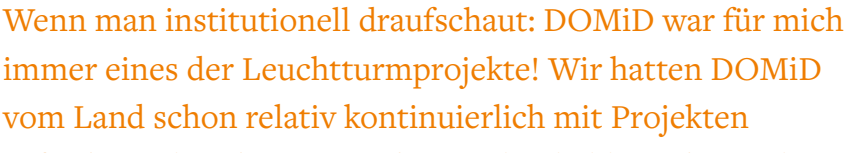


Im Jahr 2007 beauftragte das nordrhein-westfälische Landesministerium für Integration DOMiD damit, mithilfe von Landesmitteln einen Plan zu entwickeln, wie die durch das Projekt Migration stark angewachsene Sammlung systematisch für die Öffentlichkeit aufgearbeitet werden könnte. Aytaç Eryılmaz betraute damit Beate Rieple, seit 2008 Leiterin des Archiv-Bereichs bei DOMiD.

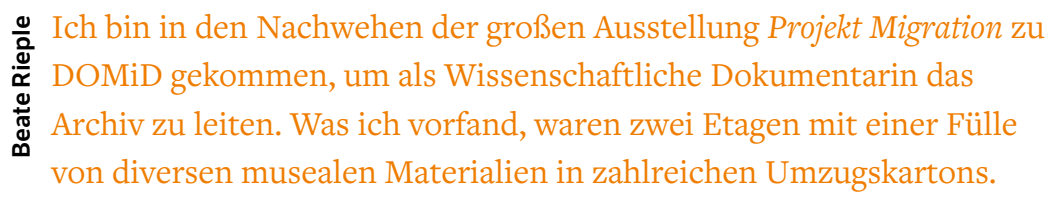
Die Büroschränke waren voll mit Objekten und Dokumenten in Archivboxen. Die Schätze im Keller habe ich erst kurz vor dem Umzug entdeckt: ein ganzer Kellerraum, vom Boden bis unter die Decke mit Möbelstücken eines Wohnheims vollgestellt. Mit einem Klemmbrett bewaffnet und einer Praktikantin im Schlepptau haben wir Objektlisten erstellt, um damit einen ersten Überblick über die Sammlung zu erhalten. Der nächste Schritt war dann, jedes einzelne Objekt auszupacken und eine Inventarnummer zu vergeben. Anschließend wurden sämtliche Materialien in bestimmte Bestandsgruppen einklassifiziert wie 3-D-Objekte, Filme, Tonaufnahmen, Einzeldokumente, Schriftgut, Plakate, Flugschriften, Zeitungen und Publikationen. All das taten wir mit dem Ziel, die Sammlung systematisch zu erschließen und der Öffentlichkeit zu präsentieren. Im Zuge des Fünf-Jahres-Plans, den wir für die Jahre 2008 bis 2012 entwickelten, hat das Ministerium dann gesehen: Bei DOMiD finden sich viele außergewöhnliche Dokumente der deutschen Migrationsgeschichte!

Auf der Grundlage der politischen Debatten begann das Land NRW, Verantwortung für das Migrationsarchiv zu übernehmen. DOMiD ist bis heute eine von nur drei Einrichtungen des Landes NRW, die vonseiten der Integrationspolitik institutionell gefördert werden: Der Landesintegrationsrat - also der Zusammenschluss aller kommunalen Integrationsräte, der früher sogenannten Ausländerbeiräte - wird institutionell gefördert; das Zentrum für Türkeistudien in Essen wird gefördert, und DOMiD als Archiv der Zuwanderungsgeschichte erhält eine institutionelle Förderung - was durchaus als Auszeichnung zu werten ist.

Mit der Förderung wurde DOMiDs Schlüsselrolle anerkannt: Das historische Gedächtnis der Migration war eine Bedingung für die Möglichkeit, ein Zugehörigkeitsgefühl zum Staats- und Gemeinwesen überhaupt erst zu entwickeln. Davon zeugt nicht zuletzt ein Antrag aus dem August 2007, der ebenfalls durch die Fraktion von 
CDU und FDP in den nordrhein-westfälischen Landtag eingebracht wurde. Unter dem Titel „Ohne Vergangenheit keine Zukunft: Erinnerungskultur gestalten - kulturelles Gedächtnis bewahren und beschützen" stellte der Landtag fest: „Wir leben in einer Zeit schneller und einschneidender gesellschaftlicher Veränderungen. [...] Der Blick auf die größeren Zusammenhänge wird immer schwieriger. Wohin soll die Reise gehen? In welcher Gesellschaft wollen wir leben? Wie kann die Welt der Zukunft gestaltet werden? Und vor allem: Was sind die Maßstäbe, mit denen wir in der Lage sind, solche Fragen zu beurteilen? Inwieweit hilft uns unser kulturelles Gedächtnis bei der Entwicklung von Perspektiven? Können die historischen Wissenschaften heute noch Orientierung bieten zwischen Vergangenheit und Zukunft? Kulturelles Gedächtnis ist kein nebensächliches Politikfeld. Ohne reflektierte Vergangenheit kann Zukunft nicht gestaltet werden. Wir in Deutschland wissen das besonders. Ein kollektives Gedächtnis ist identitätsstiftend. ${ }^{\text {"5 }}$

Parallel zu diesen Entwicklungen erhielt DOMiD aus der Kölner Stadtgesellschaft zunehmend konkrete Unterstützung. 2007 gründete sich der Förderverein DOMiD e.V., um insbesondere die Arbeit DOMiDs durch politische und finanzielle Unterstützung abzusichern. Zu den beteiligten Akteur*innen zählten die Journalistin Helga Kirchner und Jürgen Nordt, der ehemalige Leiter des Kölner Kulturamts. ${ }^{6}$ Auch politische Entscheidungsträger*innen in Köln begannen, DOMiD als möglichen Standortfaktor zu begreifen. So unterstützte die Stadt Köln - namentlich die Sozialdezernentin Marlis Bredehorst - die Institutionalisierung DOMiDs, indem sie dem Migrationsarchiv - vom Jahr 2009 an - die gesamte oberste Etage des Bezirksrathauses Ehrenfeld zur Verfügung stellte. Auf knapp 1.000 Quadratmetern hatte DOMiD nun endlich genügend Raum, sich zu entfalten. Als Geschäftsstelle und Kompetenzzentrum, vor allem aber auch zur Installation eines professionellen Depots. Hierher wurden die Objekte aus den Kellerräumen des ehemaligen Hochhauses der Deutschen Welle nun umgelagert. Mit der Übersiedlung in das Bezirksrathaus in Köln-Ehrenfeld und der institutionellen Förderung durch das Land NRW hatte DOMiD einen wichtigen Etappensieg errungen.

\footnotetext{
․ㅡㄹ Aytaç Eryllmaz hatte schon verschiedene Orte in Köln besichtigt. Da gab es aber immer nur entweder Ausstellungsfläche oder Depoträume für die Sammlung. Für ihn war aber klar, er wird nur an einen Ort umziehen, wo beides vorhanden ist. Das war das zentrale Kriterium. Und hier sind wir
}

5 https://www.landtag.nrw.de/portal/WWW/ dokumentenarchiv/ Dokument/MMD144869.pdf (aufgerufen am 10.05.2021).

6 Der Verein bestand bis 2015. Durch seine Tätigkeit und finanzielle Unterstützung konnte DOMiD zahlreiche Projekte durchführen. 
eingezogen, weil hier das Archiv sein sollte, und unten im Innenhof - wo früher ein Getränkemarkt drin war und wo sich heute ein Kinderhort befindet -, da sollte die Ausstellungsfläche sein. Dann hat man aber festgestellt, dass das Dach da unten undicht war und die Stadt Köln unter diesen Umständen das Gebäude doch nicht anmieten wollte. Als sich das herausstellte, waren wir aber schon dabei, hier ins Rathaus umzuziehen. 2010 im Januar war das. Das war sehr spannend. Wir haben dann zunächst all die 3-D-Objekte ausgepackt und sie dann nach Materialien getrennt: Kunststoff, Keramik, Glas, Metall, Elektrogeräte, Holz, Karton, Textil und Leder. In diesen ersten Wochen konnten wir die musealen Objekte näher begutachten. Zum Beispiel diesen Schuhputzerschrank aus Berlin, der besteht aus über 500 Teilen. Darunter Fläschchen mit unterschiedlichen Farbtönen, oder Cremes, die schon angetrocknet waren. Mithilfe einer Masterstudentin vom Institut für Restaurierungs- und Konservierungswissenschaft in Köln konnten Objektkonvolute dann unter sachkundiger Anleitung gereinigt, ausgedünnt und sorgfältig verpackt werden. Da muss man dann entscheiden: Schütten wir die verderblichen Inhalte der Fläschchen weg, oder füllen wir sie in Ersatzgefäße um, für kommende Ausstellungen? Oder das Elternhaus-Modell von Petros Melitopoulos, das aus Materialien wie Haferflocken oder Vogelfutter gebaut ist. Diese Materialien mussten wir separieren und in Kunststoffhüllen verpacken, damit keine Schädlinge angezogen wurden.

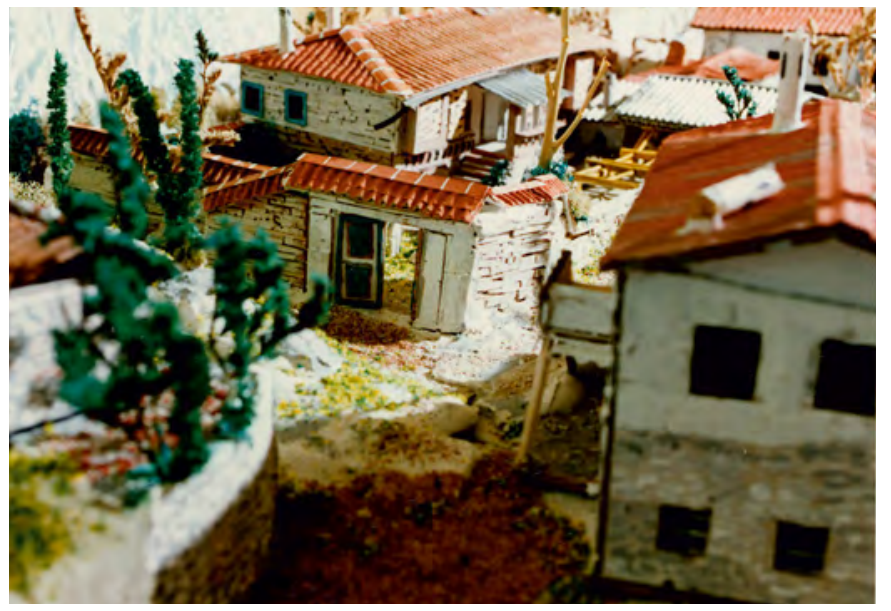

E 0089,00013 Dorfmodell Ptelea von Petros Melitopoulos, 1990er-Jahre. DOMiD-Archiv, Köln

Die Familie des Modellbauers Petros Melitopoulos stammt aus Hafsa am Schwarzen Meer. Nach der Vertreibung der Griechen aus Kleinasien 1922 wird die Familie in einem Dorf nahe der nordgriechischen Stadt Drama angesiedelt: in Ptelea. Es ist dieses Dorf, das Petros Melitopoulos Jahrzehnte später in Deutschland aus Pappkartons, Reisnudeln und Haferflocken rein aus seiner Erinnerung rekonstruierte. 